\title{
On the Robustness of Network Community Structure Under Addition of Edges
}

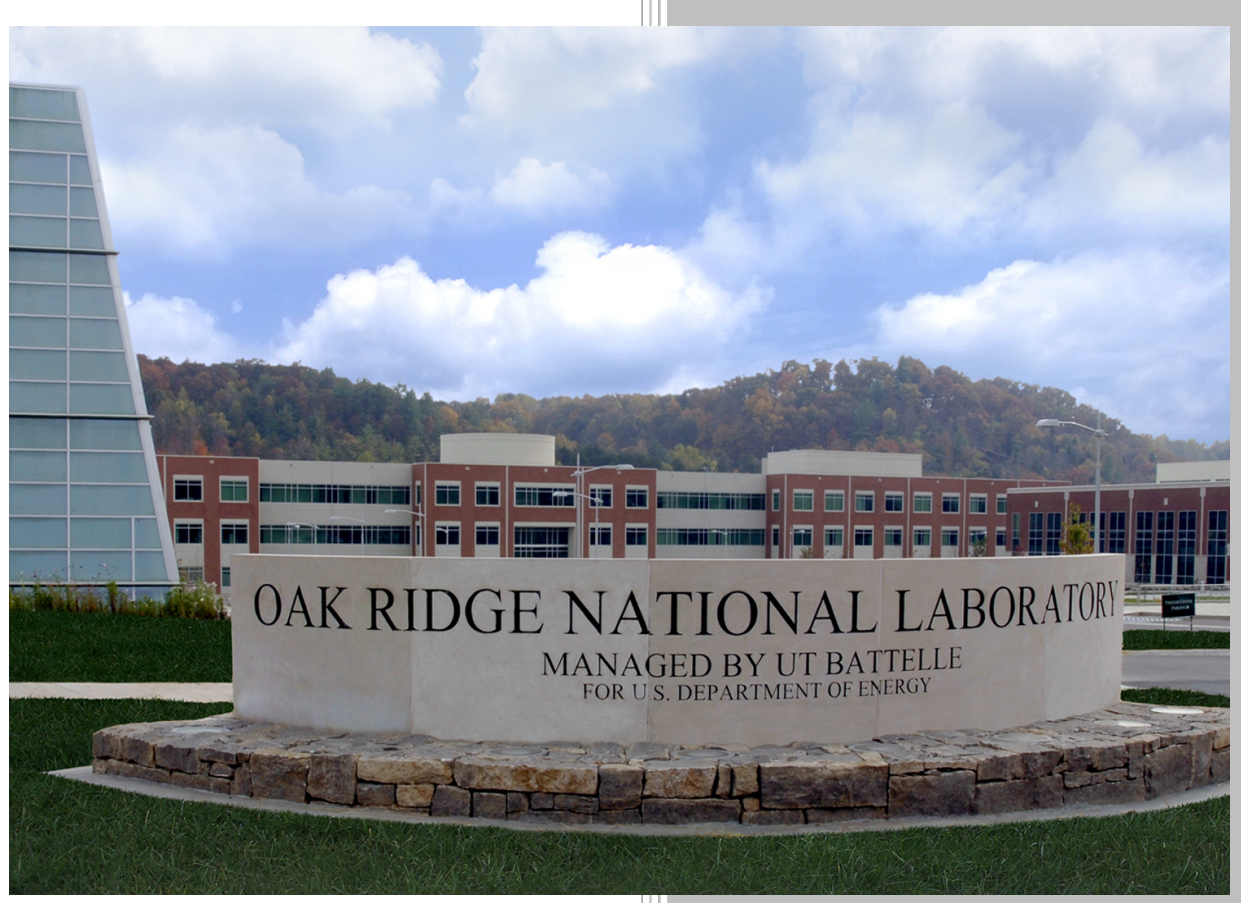

Approved for public release.

Pablo Moriano

Kalyan Perumalla Distribution is unlimited.

September 16, 2020 


\section{DOCUMENT AVAILABILITY}

Reports produced after January 1, 1996, are generally available free via US Department of Energy (DOE) SciTech Connect.

Website: http://www.osti.gov/scitech/

Reports produced before January 1, 1996, may be purchased by members of the public from the following source:

National Technical Information Service

5285 Port Royal Road

Springfield, VA 22161

Telephone: 703-605-6000 (1-800-553-6847)

TDD: 703-487-4639

Fax: 703-605-6900

E-mail: info@ntis.gov

Website: http://classic.ntis.gov/

Reports are available to DOE employees, DOE contractors, Energy Technology Data Exchange representatives, and International Nuclear Information System representatives from the following source:

Office of Scientific and Technical Information

PO Box 62

Oak Ridge, TN 37831

Telephone: 865-576-8401

Fax: 865-576-5728

E-mail: report@osti.gov

Website: http://www.osti.gov/contact.html

This report was prepared as an account of work sponsored by an agency of the United States Government. Neither the United States Government nor any agency thereof, nor any of their employees, makes any warranty, express or implied, or assumes any legal liability or responsibility for the accuracy, completeness, or usefulness of any information, apparatus, product, or process disclosed, or represents that its use would not infringe privately owned rights. Reference herein to any specific commercial product, process, or service by trade name, trademark, manufacturer, or otherwise, does not necessarily constitute or imply its endorsement, recommendation, or favoring by the United States Government or any agency thereof. The views and opinions of authors expressed herein do not necessarily state or reflect those of the United States Government or any agency thereof. 
Computer Science and Mathematics Division

On the Robustness of Network Community Structure Under Addition of Edges

Pablo Moriano

Kalyan Perumalla

Date Published: September 16, 2020

Prepared by

OAK RIDGE NATIONAL LABORATORY

Oak Ridge, TN 37831-6283

managed by

UT-Battelle, LLC

for the

US DEPARTMENT OF ENERGY

under contract DE-AC05-00OR22725 



\section{CONTENTS}

LIST OF FIGURES $\ldots \ldots \ldots \ldots \ldots \ldots \ldots \ldots \ldots \ldots \ldots \ldots$

LIST OF TABLES $\ldots \ldots \ldots \ldots \ldots \ldots \ldots \ldots \ldots \ldots \ldots \ldots \ldots \ldots$ vii

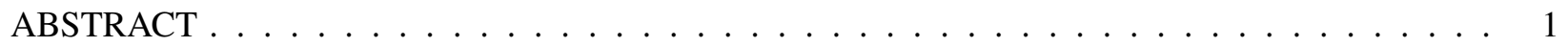

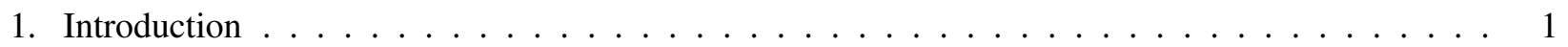

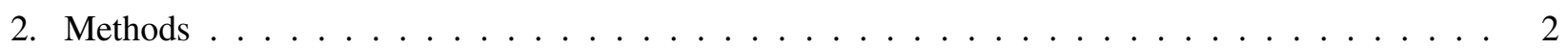

2.1 Network Benchmarks . . . . . . . . . . . . . . . . . . . . 3

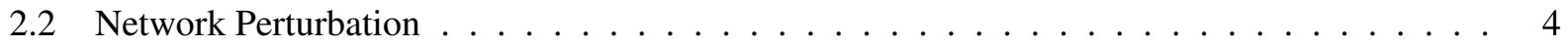

2.3 Community Detection Algorithms $\ldots \ldots \ldots \ldots \ldots \ldots$

2.4 Similarity of Community Structure $\ldots \ldots \ldots \ldots \ldots \ldots \ldots$

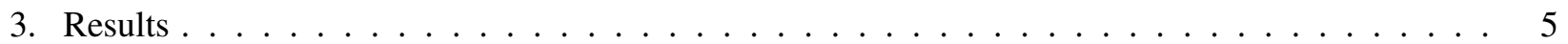

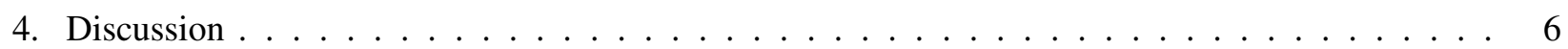

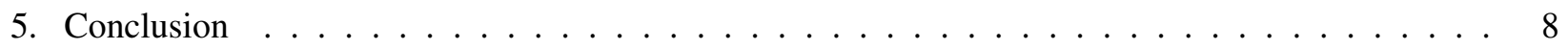





\section{LIST OF FIGURES}

1 Variation in the NMI as a function of the number of added edges. $\quad \ldots \ldots \ldots$

2 Variation in the standard deviation as a function of the number of added edges. . . . . . . 7 



\section{LIST OF TABLES}

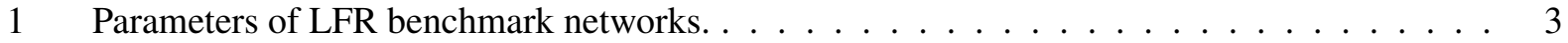





\begin{abstract}
Communities represent important functional modules in networked systems. A key goal in preserving such communities is understanding their robustness under perturbations. Previous research has studied the impact of node removals and edge removals on the community structure. However, the impact of edge additions on the robustness of the community structure is relatively unknown. Edge additions or false positive edges may simulate measurement errors or external exceptional events that threaten the functionality of networked systems. Here, we study the impact of edge additions on the community structure using Lancichinetti-Fortunato-Radicchi (LFR) benchmark networks. We show that, for a fixed network size, the impact of edge additions is greater on networks with initially weak community structure than on networks with strongly clustered structures. In addition, we find that the perception of the impact is also dependent on the community detection algorithm used to uncover communities. In particular, we found that modularity-based methods such as Leiden and Louvain are less affected than information-theoretic and message passing-based methods such as Infomap and Label Propagation. Our results demonstrate that edge addition can (a) significantly impact the community structure of networks based on their initial conditions, and (b) the perception of the impact is dependent on the community detection algorithm used. We describe limitations, open challenges, and how this methodology can inform the design of resilient networked systems under edge additions.
\end{abstract}

\title{
1. INTRODUCTION
}

Many complex systems are usually represented by networks (Amaral and Ottino, 2004). Networks are useful representations because they capture interactions among the elements of these complex systems and aid us in understanding their structure and function (Newman, 2003). Networked representations have been found useful in uncoupling a wide range of sources of complexity among different applications, including, percolation (Albert et al., 2000), spreading processes (Newman et al., 2002), diffusion (Weng et al., 2013) synchronization (Wang et al., 2007), signaling (Wang et al., 2012b), and game dynamics (Moriano and Finke, 2012), among others. Among the most commonly studied properties of networks, the property of community structure has emerged as key to understand and explain many aspects of complex systems. Communities usually represent important functional modules in networked systems (Lancichinetti et al., 2010).

Communities serve to capture a mesoscale representation of networks. Communities are abstractions that group multiple nodes of the network that have similar properties. Nominally, this is done by grouping nodes with a higher intra-community density than inter-community density. Communities have been found to reflect important functional modules in different kinds of complex systems, including the brain (Sporns and Betzel, 2016), society (Watts et al., 2002), and networked engineering systems (Leskovec et al., 2008). Thus the development of methods to automatically uncover communities has been an active area of research over the last few years (Fortunato and Hric, 2016).

The identification of communities within a networked system has been shown to be an important and open challenge (Fortunato, 2010). Additionally, there is an increasing interest in understanding the limits of the robustness of the identified community structure. This is because maintaining the functionality of networked systems is heavily dependent on maintaining their community representation. In other words, community structure affects the maintenance of their functional clusters (Wang et al., 2017). A robust community structure should keep similar community assignments to nodes before and after perturbations. 
To that end, different perturbation mechanisms have been tested, most of which are based on node and edge deletion (Amancio et al., 2015; Wang and Liu, 2018), and edge rewiring (Karrer et al., 2008). These perturbation mechanisms are usually used as a proxy of unpredictable failures or attacks.

Here, we focus on understanding the limits of the robustness of community structure under the addition of edges. Perturbing a network by adding new edges would be one way to simulate the measurement errors for a real networked system where nodes are erroneously connected among themselves, but in reality are not connected. In other words, the added edges could represent false positive edges (Borgatti et al., 2006; Wang et al., 2012a). This has important implications, for instance, in social networks, where declared contacts may, in fact, not represent real relationships (Choudhury et al., 2010). The addition of edges may also simulate the impact of exceptional events on social communications networks, such as Twitter. During exceptional events, a high volume of interactions is expected (Moriano et al., 2019). However, to the best of our knowledge, the impact of exceptional events on the robustness of the community structure and its implications has not yet been thoroughly studied.

The contributions of this research are summarized as follows. First, we show the impact of adding random edges on the robustness of the community structure of networks by controlling their size. We build on the previous work of (Wang and Liu, 2018) that quantified the impact of removing edges on the robustness of the community structure. By contrast, we systematically add edges to synthetically generated networks conforming to power law degree distribution and community size using LFR network benchmarks (Lancichinetti et al., 2008). We compare changes in the the robustness under different community structure strengths, using state-of-the-art community detection algorithms. We observe that for the addition of edges, the stronger the community structure is, the more robust also is the community structure. These results also indicate that the degree of robustness of the community structure is dependent on the specific community detection algorithm used. In particular, modularity-based algorithms (e.g., Leiden (Traag et al., 2019) and Louvain (Blondel et al., 2008)) smoothen the impact of the addition of edges, while information-theoretic (e.g., Infomap (Rosvall and Bergstrom, 2008)) and message passing-based (e.g., Label Propagation (Raghavan et al., 2007)) measures are heavily impacted by edge additions. In addition, we show that variations of robustness when adding edges at different proportions are negligible for Leiden and Louvain but not for Infomap and Label Propagation. This indicates that the perception on the impact on the community structure is biased with respect to the nature of the community detection algorithm applied.

The rest of this paper is organized as follows. In Sec. 2., we detail the experimental setup of this research, including LFR network benchmarks, the network perturbation mechanism, community detection algorithms, and the similarity measure we used to compare community structures. Detailed experimental results are presented in Sec. 3.. This includes results using networks of fixed size. In Sec. 4., we discuss the implications of our results, including their limitations. Finally, Sec. 5. presents concluding remarks and areas for future research.

\section{METHODS}

In this section, we describe in detail the procedure to generate the synthetic network benchmarks (Sec. 2.1), the procedure to perturb the generated networks (Sec. 2.2), the community detection algorithms we used (Sec. 2.3), and the quantification of similarities in the community structure (Sec. 2.4). 


\subsection{NETWORK BENCHMARKS}

There are two options to compare the performance of community detection methods. We can use either empirical networks with labeled information about community assignments (metadata groups) or synthetic network benchmarks with known community memberships (ground truth). Among the former, previous research has relied on networks such as the Zachary's karate club (Zachary, 1977), the Lusseau's dolphins's network (Lusseau, 2003), and the college football network (Girvan and Newman, 2002). Despite the increasing availability of empirical networks with metadata groups, the work in (Hric et al., 2014) shows that there is a mismatch between topological and metadata groups that is consistent among various community detection methods. Among the latter, the predominant network generators include the Girvan-Newman (GN) benchmark (Girvan and Newman, 2002) and the LFR benchmark introduced by Lancichinetti et al. (Lancichinetti et al., 2008). The GN benchmark consists of 128 nodes clustered into 4 equal-sized communities. In the GN benchmark, nodes have approximately the same degree and the number of edges within communities exceeds the number of edges across communities. The GN benchmark, however generates networks with small sizes and does not exhibit common real-world network topological properties such as power law degree distributions (Barabási and Albert, 1999; Newman, 2003) and community size (Guimerà et al., 2003; Clauset et al., 2004) distributions. The LFR benchmark introduces the ability to control the generation of networks with power law degree and community size distributions commonly found in empirical networks (Lancichinetti et al., 2008). To do so, the LFR benchmark allows control on the degree of membership of nodes to communities through the mixing parameter $\mu$. In particular, $\mu$ controls the fraction of node's edges that are external to its assigned community. Thus it becomes more challenging for community detection algorithms to correctly detect communities with larger values of $\mu$. In general, it is expected that for $\mu>0.5$, nodes have fewer connections within the community than with the rest of the network (Yang et al., 2016). In our present work, we use the LFR benchmark for the generation of synthetic networks with known community membership (ground truth). We focus on undirected, unweighted networks represented by graphs. A graph is denoted by $G=(V, E)$, where $V=\{1,2, \ldots, N\}$ is the set of $N$ nodes and $E=\left\{e_{i j} \mid i, j \in V\right\}$ is the set of $M$ edges. Let $C=\left\{c_{1}, c_{2}, \ldots, c_{N}\right\}$ denotes a partition of graph $G$ indicating the community membership of each node. In other words, $c_{i}$ ans $c_{j}$ have the same value if both nodes $i$ and $j$ belong to the same community. We focus on non-overlapping communities given that the majority of community detection algorithms are designed for this type of networks. Table 1 shows the set of parameter values that we used in the generation of the LFR benchmark networks.

Table 1. Parameters of LFR benchmark networks.

\begin{tabular}{|l|c|}
\hline \multicolumn{1}{|c|}{ Parameter } & Value \\
\hline \hline Number of nodes $N$ & 1000 \\
Maximum degree $k_{\max }$ & $0.1 N$ \\
Maximum community size $s_{\max }$ & $0.1 N$ \\
Average degree $\langle k\rangle$ & 10 \\
Degree distribution exponent $\alpha$ & -2 \\
Community size distribution exponent $\beta$ & -1 \\
Mixing coefficient $\mu$ & {$[0.01,0.5]$} \\
\hline
\end{tabular}




\subsection{NETWORK PERTURBATION}

Here we focus on comparing networks that have the same number of nodes. We only perturb the generated networks by adding edges. Edge addition refers to the addition of edges not present in the generated LFR benchmark networks. In particular, we randomly add new edges by connecting two previously unconnected nodes. Let $G^{\prime}=\left(V, E^{\prime}\right)$, where $E^{\prime}=\left\{e_{i j}^{\prime} \mid i, j \in V\right\}$ is the perturbed network. The number of added edges constitute up to $\left|E^{\prime}\right|=10 M$ random edges.

\subsection{COMMUNITY DETECTION ALGORITHMS}

Community detection is the task of assigning nodes to communities based on the similarity of their topological properties (Fortunato, 2010). Community detection performs grouping of nodes inside the same community if the nodes are highly connected among themselves (high intra-community density) but loosely connected with nodes of other communities (low inter-community density). In this work, we use state-of-the-art community detection algorithms that scale to networks containing up to millions of nodes. We use their undirected, unweighted implementations to remain consistent with the generated LFR network benchmarks. We use the lowest hierarchical level of communities returned by the algorithms. Below is the list of the algorithms we used, in alphabetical order.

Infomap. The Infomap algorithm was proposed by Rosvall et al. (Rosvall and Bergstrom, 2008). This algorithm is based on the principles of information theory. Infomap finds optimal communities by maximizing an objective function called Minimum Description Length (Rosvall and Bergstrom, 2007). This is equivalent to finding a description of minimum information of a random walk on the graph. The computational complexity of Infomap is $O(M)$ (Delvenne et al., 2013). We use the default parameters of the implementation at (A. Lancichinetti).

Label Propagation. The label propagation algorithm was proposed by Raghavan et al. (Raghavan et al., 2007). Label propagation does not optimize a predefined objective function. Instead it relies on an iterative process in which each node in the graph adopts the label that is more commonly found among its neighbors (assigned uniquely at the beginning). The process stops when every node in the graph has the most common label among its neighbors. The computational complexity of the label propagation algorithm is $O(M)$ (Raghavan et al., 2007). We use the default parameters of its publicly available implementation (A. Lancichinetti).

Leiden. The Leiden algorithm was proposed by Traag et al. (Traag et al., 2019). This algorithm tries to optimize a quality partition function defined either by Newman-Girvan (Newman and Girvan, 2004) or the Constant Potts Model (Traag et al., 2011). It requires three steps. First, the algorithm moves nodes across communities aiming to optimize the partition. Secondly, the algorithm refines a previously determined partition by splitting a community into multiple communities when they become badly connected after node movements. Finally, the algorithm performs aggregation of the network based on the redefined partition. These steps are repeated until no further improvements can be made. The computational complexity of Leiden is $O(N \log N)$ (Traag et al., 2019). We use the Newman-Girvan modularity function, a resolution parameter of 1.0, with 10 random starts, and 10 iterations per random start. We use the publicly available implementation of this algorithm (Traag, 2018). 
Louvain. The Louvain algorithm was proposed by Blondel et al. (Blondel et al., 2008). This algorithm attempts to optimize the modularity of network partition as defined by

Newman-Girvan (Newman and Girvan, 2004). This procedure requires two steps. Fist, the algorithm optimizes modularity locally based on each node assignment. Second, the algorithm aggregates nodes in the same community to form super-nodes. These steps are repeated until a maximum of modularity is achieved. The computational complexity of Louvain is $O(N \log N)$ (Xie and Szymanski, 2011). We use 10 modularity maximization iterations (the default value) and use the partition that produces the greatest modularity. We use a publicly available implementation of this algorithm (A. Lancichinetti).

\subsection{SIMILARITY OF COMMUNITY STRUCTURE}

Let $C^{\prime}=\left\{c_{1}^{\prime}, c_{2}^{\prime}, \ldots, c_{N}^{\prime}\right\}$ be the partition of the graph $G^{\prime}$ obtained by a community detection algorithm. We quantify the similarity between the ground truth partition $C$ and the detected partition $C^{\prime}$ using the normalized mutual information (NMI) (Danon et al., 2005), normalized by the arithmetic mean. We use the scikit-learn implementation of NMI. This measure of similarity borrows information-theoretic ideas (Vinh et al., 2010) and has been used extensively when comparing community detection algorithms performance (Lancichinetti and Fortunato, 2009). More formally, let the Shannon entropy of $C$ be $H(C)=-\sum_{c} P(c) \log P(c)$, where $P(c)$ is the probability that a randomly selected node is assigned to the community $c$. Similarly, let the Shannon entropy of $C^{\prime}$ be $H\left(C^{\prime}\right)=-\sum_{c^{\prime}} P\left(c^{\prime}\right) \log P\left(c^{\prime}\right)$ and $H\left(C, C^{\prime}\right)=-\sum_{c} \sum_{c^{\prime}} P\left(c, c^{\prime}\right) \log P\left(c, c^{\prime}\right)$, where $P\left(c, c^{\prime}\right)$ is the joint probability that a randomly selected node is assigned to the community $c$ by $C$ and to the community $c^{\prime}$ by $C^{\prime}$. The mutual information of $C$ and $C^{\prime}$ relies on the entropies of $C$ and $C^{\prime}$ and is defined as $I\left(C, C^{\prime}\right)=H(C)+H\left(C^{\prime}\right)-H\left(C, C^{\prime}\right)$. This results in the following equation.

$$
I\left(C, C^{\prime}\right)=\sum_{c} \sum_{c^{\prime}} P\left(c, c^{\prime}\right) \log \frac{P\left(c, c^{\prime}\right)}{P(c) P\left(c^{\prime}\right)}
$$

The mutual information between $C$ and $C^{\prime}$ quantifies the overlap between $C$ and $C^{\prime}$. More precisely, it represents how much we learn about $C$ from knowing $C^{\prime}$ (and vice versa). To obtain a normalized value between 0 and 1, we use the definition of the NMI (Danon et al., 2005) as

$$
\operatorname{NMI}\left(C, C^{\prime}\right)=\frac{2 I\left(C, C^{\prime}\right)}{\sqrt{H(C) H\left(C^{\prime}\right)}} .
$$

Perfectly similar partitions produce NMI values of unity, while perfectly dissimilar partitions produce NMI values equal to zero.

\section{RESULTS}

Here we present our results on the robustness of the community structure under the random addition of edges. We quantify the impact of random edge addition on the robustness of the community structure by computing the similarity of the partitions $C$ and $C^{\prime}$. In the subsequent figures, each data point represents statistics computed over 20 realizations of random edge additions.

In this work we focus on the impact of adding random edges to the community structure of networks of fixed size (Sec. 3.). We show that the magnitude and rate of the impact depends (1) on the strength of the 
initial community structure (captured through the mixing parameter $\mu$ ) and (2) the algorithm used for community detection.

\section{Constant Network Size}

To investigate how the random addition of edges impacts the community structure, we compute the NMI for different values of added edges. Note that we repeat the same procedure varying the value of the mixing parameter $\mu$. We focus on LFR benchmark networks of size $N=1000$ (following the remaining parameter values in Table 1).

We find that, for a fixed network size, the magnitude and speed of the impact depends on $\mu$ and the community detection algorithm. With respect to $\mu$, the lower the value, the more robust is the community structure (and vice versa). With respect to the community detection method, both information theory-based (Infomap) and message passing-based (Label Propagation) algorithms are less robust to the random addition of edges than their counterpart based on modularity optimization (Leiden and Louvain).

Fig. 1 shows the effect of $\mu$ and the community detection algorithm on the robustness measured through the average NMI. Regarding $\mu$, networks with stronger community structure (lower values of $\mu$ ) have a slower decay in the NMI, i.e., it is necessary to add more edges in order to reduce the NMI to a similar value to one of a network with a higher value of $\mu$. Regarding community detection algorithms, Infomap and Label Propagation show a sharp decrease in the robustness at lower values of added edges. In particular, when added 3 and 2 times the number of original edges respectively, the NMI tends to 0, i.e., highly dissimilar communities. This is not the case for Leiden and Infomap that with about 10 times the number of edges added still show traits of similar partitions depending on the value of $\mu$.

Fig. 2 shows the effect of $\mu$ and the community detection algorithm on the robustness variation measured through the standard deviation of the NMI. In particular, for Infomap and Label Propagation, networks with stronger communities (lower values of $\mu$ ) tend to have higher variability than their counterpart. Interestingly, for Leiden and Louvain, the variation is about one order of magnitude lower than that for Infomap and Label Propagation. For Leiden and Louvain, the variations do not show an increasing or decreasing pattern with respect to $\mu$.

\section{DISCUSSION}

Here, we study the impact of the random addition of edges on the robustness of the community structure of networks. We quantify the robustness of the community structure by computing the NMI between the partition of the original network (ground truth) and the partition found by four different community detection algorithms, namely, Infomap, Label Propagation, Leiden, and Louvain. To accomplish this, we use LFR benchmark networks as proxies of network substrates and then add random edges that did not exist in the original networks. We add a number of edges proportional to the original number of edges. We find that, for a fixed size network, the robustness of the community structure depends on the strength of the community structure of the original network and the community detection method used.

These results suggest that the robustness of a network against the random addition of edges depends heavily on the strength of the initial partition. First, we find that stronger community partitions tend to be more robust when adding random edges. Second, we find that the choice of the community detection algorithm has an impact in both the severity and the rate of decrease of the robustness. Infomap and Label 
(a) Infomap

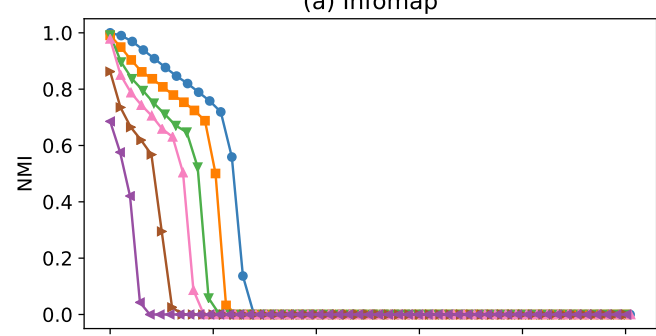

(c) Leiden

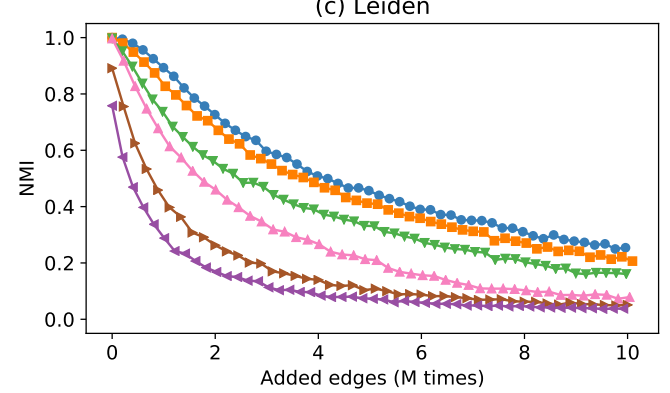

(b) Label Propagation

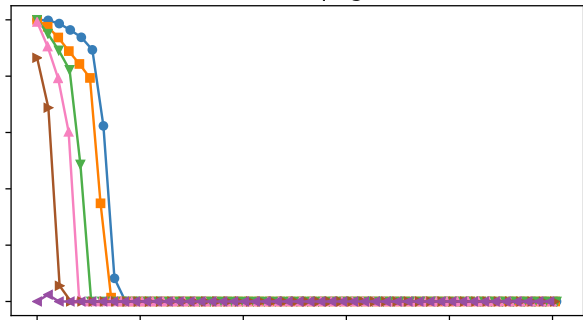

(d) Louvain

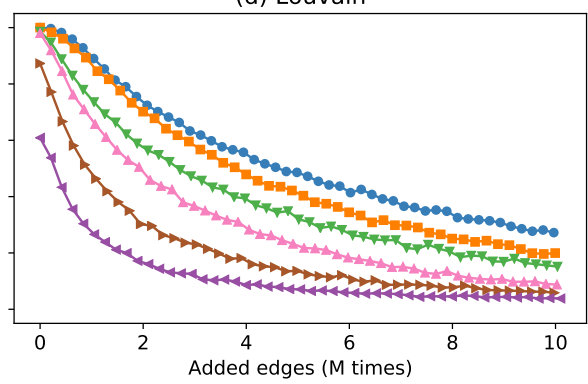

Figure 1. Variation in the NMI as a function of the number of added edges.

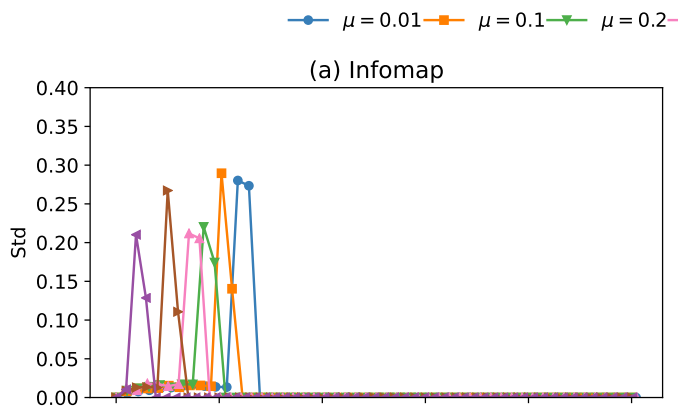

$\mu=0.3 \longrightarrow \mu=0.4 \longrightarrow \mu=0.5$
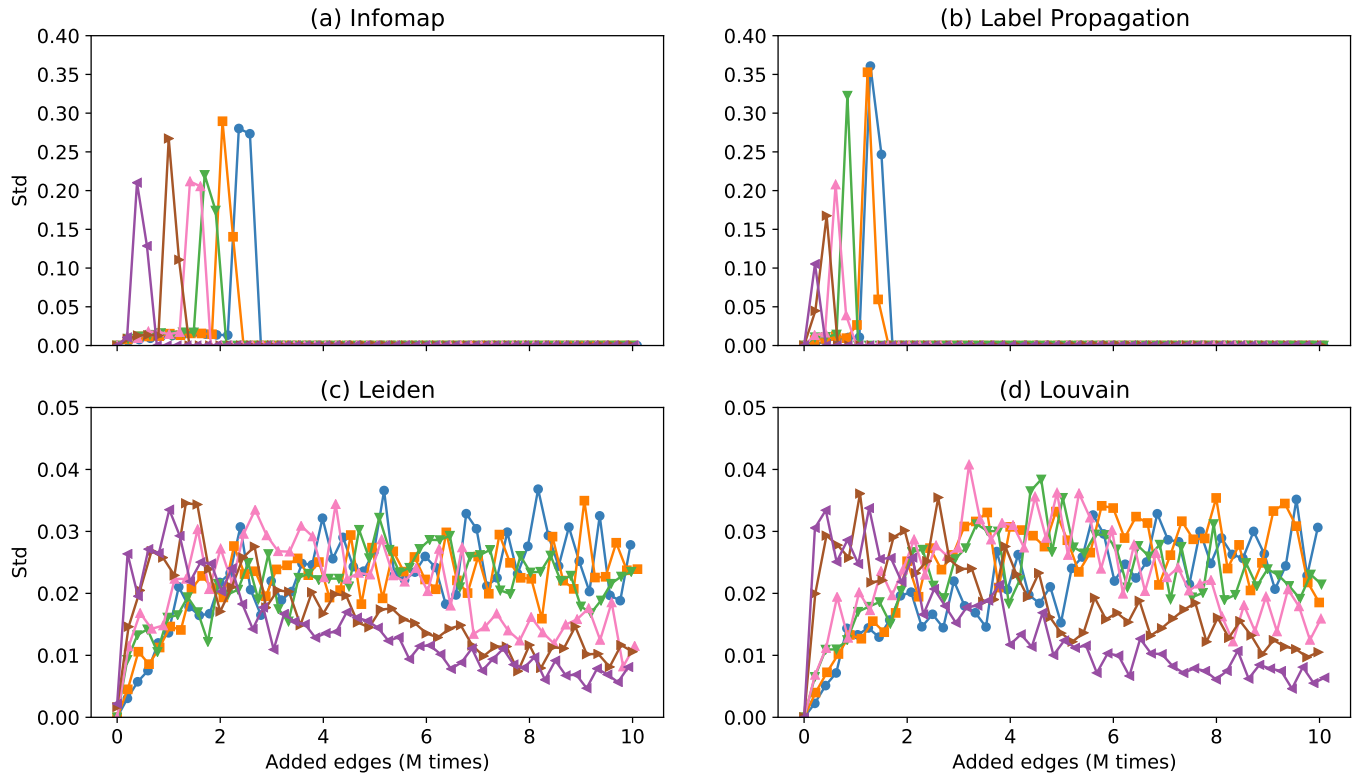

Figure 2. Variation in the standard deviation as a function of the number of added edges. 
Propagation tend to sharply decrease their robustness, indicating that they hardly resemble the original partition when there is an increasing amount of noise added to the system. Leiden and Louvain are found to be more robust under the increasing number of added edges even when the initial partition is not very well defined.

The scripts used in this work will be made available for reproducibility purposes.

To the best of our knowledge, the results from this research show for the first time a systematic evidence on the impact of random addition of edges on the robustness of the community structure.

The following are some of the limitations of our study.

Emphasis on LFR network benchmarks: We use LFR network benchmarks as a substrate and then add random edges. LFR network benchmarks allow the generation of networks with both power law degree and community size distributions. We acknowledge that although having both power law degree and community size distributions are desirable properties, they many not fully resemble all the properties found in empirical networks (Newman, 2003). Additional stochastic network generators that include embedded community structure may be helpful in addressing similar questions as in this work by focusing on additional network properties (Funke and Becker, 2019).

Constant average degree: In our experiments, we keep constant the average degree of the nodes $\langle k\rangle$. We acknowledge, however, that the density of the initial connections may influence the task of community detection, making it even harder for some algorithms. Further studies on this matter can be implemented on top of our proposed methodology.

Random addition of edges: We add non-existing edges randomly. We smooth the impact of this strategy by running multiple realizations of the same task. However, we notice that the strategy of adding random edges can be targeted to emphasize the addition of certain types of edges, such as inter-community or intra-community edges. This may have a completely different impact on the robustness of the community structure.

\section{CONCLUSION}

In this research, we have shown that the process of adding edges to a defined community structure has an impact its robustness. To ascertain this fact in experiments, we use LFR network benchmarks and show that the degree at which the robustness is affected depends heavily on the strength of the initial partition, controlled by the mixing parameter $\mu$. Moreover, among different community detection methods, the impact at which the robustness is affected depends on the community detection algorithm employed. We have shown that the perception is more susceptible for Infomap and Label Propagation than for Leiden and Louvain.

Future work in this area includes studying the impact of the addition of edges to networks generated with different average degrees. Additional work includes the study of the robustness of the community detection under different stochastic block models besides LFR network benchmark and empirical networks. 


\section{References}

A. Lancichinetti. Andrea Lancichinetti's homepage. URL https://sites.google.com/site/andrealancichinetti/. Date last accessed September 11, 2020.

R. Albert, H. Jeong, and A.-L. Barabási. Error and attack tolerance of complex networks. Nature, 406 (6794):378, 2000.

D. R. Amancio, O. N. Oliveira Jr, and L. da F Costa. Robustness of community structure to node removal. Journal of Statistical Mechanics: Theory and Experiment, 2015(3):P03003, 2015.

L. A. N. Amaral and J. M. Ottino. Complex networks: Augmenting the framework for the study of complex systems. Eur. Phys. J. B, 38(2):147-162, 2004.

A.-L. Barabási and R. Albert. Emergence of scaling in random networks. Science, 286(5439):509-512, 1999.

V. D. Blondel, J.-L. Guillaume, R. Lambiotte, and E. Lefebvre. Fast unfolding of communities in large networks. J. Stat. Mech. Theory Exp, 2008(10):P10008, 2008.

S. P. Borgatti, K. M. Carley, and D. Krackhardt. On the robustness of centrality measures under conditions of imperfect data. Social Networks, 28(2):124-136, 2006.

M. De Choudhury, W. A. Mason, J. M. Hofman, and D. J. Watts. Inferring relevant social networks from interpersonal communication. In Proceedings of the 19th international conference on World wide web, pages 301-310, 2010.

A. Clauset, M. E. J. Newman, and C. Moore. Finding community structure in very large networks. Phys. Rev. E, 70(6):066111, 2004.

L. Danon, A. Diaz-Guilera, J. Duch, and A. Arenas. Comparing community structure identification. Journal of Statistical Mechanics: Theory and Experiment, 2005(09):P09008, 2005.

J. C. Delvenne, M. T. Schaub, S. N. Yaliraki, M. Barahona, A. Mukherjee, M. Choudhury, F. Peruani, N. Ganguly, and B. Mitra. Dynamics on and of complex networks, 2013.

S. Fortunato. Community detection in graphs. Phys. Rep., 486(3-5):75-174, 2010.

S. Fortunato and D. Hric. Community detection in networks: A user guide. Physics reports, 659:1-44, 2016.

T. Funke and T. Becker. Stochastic block models: A comparison of variants and inference methods. PLoS One, 14(4):e0215296, 2019.

M. Girvan and M. E. J. Newman. Community structure in social and biological networks. Proc. Natl. Acad. Sci. U.S.A., 99(12):7821-7826, 2002.

R. Guimerà, L. Danon, A. Díaz-Guilera, F. Giralt, and A. Arenas. Self-similar community structure in a network of human interactions. Phys. Rev. E, 68:065103, 2003. 
D. Hric, R. K. Darst, and S. Fortunato. Community detection in networks: Structural communities versus ground truth. Phys. Rev. E, 90(6):062805, 2014.

B. Karrer, E. Levina, and M. E. J. Newman. Robustness of community structure in networks. Phys. Rev. E, 77:046119, 2008.

A. Lancichinetti and S. Fortunato. Community detection algorithms: A comparative analysis. Phys. Rev. E, 80:056117, 2009.

A. Lancichinetti, S. Fortunato, and F. Radicchi. Benchmark graphs for testing community detection algorithms. Phys. Rev. E, 78:046110, 2008.

A. Lancichinetti, M. Kivelä, J. Saramäki, and S. Fortunato. Characterizing the Community Structure of Complex Networks. PLOS ONE, 5(8):1-8, 2010.

J. Leskovec, K. J. Lang, A. Dasgupta, and M. W. Mahoney. Statistical properties of community structure in large social and information networks. In Proceedings of the 17th international conference on World Wide Web, pages 695-704, 2008.

D. Lusseau. The emergent properties of a dolphin social network. Proceedings of the Royal Society of London. Series B: Biological Sciences, 270:S186-S188, 2003.

P. Moriano and J. Finke. Power-law weighted networks from local attachments. Europhysics Letters, 99 (1):18002, 2012.

P. Moriano, J. Finke, and Y.-Y Ahn. Community-Based Event Detection in Temporal Networks. Sci. Rep., 9(1):4358, 2019.

M. E. J. Newman. The structure and function of complex networks. SIAM Rev., 45(2):167-256, 2003.

M. E. J. Newman and M. Girvan. Finding and evaluating community structure in networks. Phys. Rev. E, 69:026113, Feb 2004.

M. E. J. Newman, S. Forrest, and J. Balthrop. Email networks and the spread of computer viruses. Phys. Rev. E, 66:035101, Sep 2002.

U. N. Raghavan, R. Albert, , and S. Kumara. Near linear time algorithm to detect community structures in large-scale networks. Phys. Rev. E, 76(3):036106, 2007.

M. Rosvall and C. T. Bergstrom. An information-theoretic framework for resolving community structure in complex networks. Proceedings of the National Academy of Sciences, 104(18):7327-7331, 2007.

M. Rosvall and C. T. Bergstrom. Maps of random walks on complex networks reveal community structure. Proc. Natl. Acad. Sci. U.S.A., 105(4):1118-1123, 2008.

O. Sporns and R. F. Betzel. Modular brain networks. Annual review of psychology, 67:613-640, 2016.

V. A. Traag. networkanalysis. GitHub, 2018. URL https://github.com/CWTSLeiden/networkanalysis. b9c4022164a5936fc3a623ccd22d9b2d241fa608. 
V. A. Traag, P. Van Dooren, and Y. Nesterov. Narrow scope for resolution-limit-free community detection. Phys. Rev. E, 84(1):016114, 2011.

V. A. Traag, L. Waltman, and N. J. van Eck. From louvain to leiden: guaranteeing well-connected communities. Sci. Rep., 9(1):1-12, 2019.

N. X. Vinh, J. Epps, and J. Bailey. Information theoretic measures for clusterings comparison: Variants, properties, normalization and correction for chance. The Journal of Machine Learning Research, 11: 2837-2854, 2010.

D. J. Wang, X. Shi, D. A. McFarland, and J. Leskovec. Measurement error in network data: A re-classification. Social Networks, 34(4):396-409, 2012a.

R.-S. Wang, A. Saadatpour, and R. Albert. Boolean modeling in systems biology: An overview of methodology and applications. Physical Biology, 9(5):055001, 2012b.

S. Wang and J. Liu. Constructing robust community structure against edge-based attacks. IEEE Systems Journal, 13(1):582-592, 2018.

S. Wang, J. Liu, and X. Wangåå. Mitigation of attacks and errors on community structure in complex networks. Journal of Statistical Mechanics: Theory and Experiment, 2017(4):043405, 2017.

X. Wang, Y.-C. Lai, and C. H. Lai. Enhancing synchronization based on complex gradient networks. Phys. Rev. E, 75:056205, 2007.

D. J. Watts, P. S. Dodds, and M. E. J. Newman. Identity and search in social networks. Science, 296(5571): 1302-1305, 2002.

L. Weng, F. Menczer, and Y.-Y. Ahn. Virality Prediction and Community Structure in Social Networks. Sci. Rep., 3(1):2522, 2013.

J. Xie and B. K. Szymanski. Community detection using a neighborhood strength driven label propagation algorithm. In IEEE Network Science Workshop, pages 188-195, 2011.

Z. Yang, R. Algesheimer, and C. J. Tessone. A Comparative Analysis of Community Detection Algorithms on Artificial Networks. Sci. Rep., 6:30750, 2016.

W. W. Zachary. An information flow model for conflict and fission in small groups. Journal of anthropological research, 33(4):452-473, 1977. 